\title{
Dual spinal cord injury: a case of acute traumatic T9 AIS A spinal cord injury complicated by transverse myelitis
}

\author{
Tyler M. Estes ${ }^{1} \cdot$ Esther Tompkins $^{2} \cdot$ Noel E. Pristas ${ }^{1} \cdot$ Rani H. Lindberg ${ }^{1}$
}

Received: 12 June 2020 / Revised: 12 July 2020 / Accepted: 11 August 2020

(c) International Spinal Cord Society 2020

\begin{abstract}
Introduction While the general principles of treatment of TM are agreed upon, the rarity of the disease has made determining its clinical course and etiology quite difficult. This report aims to inform these debates by demonstrating the susceptibility of at least one individual to transverse myelitis following an acute SCI.

Case presentation This case report follows the clinical course of a 17 year old. Caucasian female that contracted transverse myelitis (TM) following an acute traumatic spinal cord injury. This patient originally presented as a T9 AIS A traumatic SCI, and was then re-graded as C6 AIS A SCI following diagnosis of TM. She was later seen in clinic for 6-month follow-up and displayed post-trauma baseline scores for both strength and sensation, indicating nearly complete recovery.

Discussion In addition, this report demonstrates the importance of adequate clinical management of TM in practical terms of patient performance on clinical exams. This report also adds to the existing body of evidence suggesting early intervention is associated with better functional outcomes.
\end{abstract}

\section{Introduction}

Transverse myelitis is a relatively uncommon disease and as such, there are significant knowledge gaps in the literature regarding its etiology and clinical course. While the general principles of patient care are agreed upon, including the necessity of rapid diagnosis and treatment with high dose steroids [1-5], there is much debate regarding causation and disease progression [4-7]. This case report aims to add to the existing body of knowledge on several of these fronts by documenting the course of a rather peculiar case of TM. This case also illustrates recommended clinical decisionmaking and effective treatment for the disease.

Tyler M. Estes

TylerMEstes@gmail.com

1 Department of Physical Medicine and Rehabilitation, University of Arkansas for Medical Sciences, Little Rock, AR, USA

2 Department of Physical Medicine and Rehabilitation, University of Arkansas for Medical Sciences, Arkansas Children's Hospital, Little Rock, AR, USA

\section{Case diagnosis}

Acute traumatic T9 AIS A spinal cord injury complicated by transverse myelitis.

\section{Case presentation}

An otherwise healthy 17 year old Caucasian female underwent an uncomplicated T8 to T12 fusion after a motor vehicle accident resulted in a T9 AIS A spinal cord contusion (Fig. 1). Two days following the procedure, the patient was evaluated by the rehabilitation team and accepted for inpatient rehabilitation admission. ASIA motor exam was limited due to pain but sensory exam was consistent with a complete injury at the T9 level. Due to sustaining an open fracture, the patient was placed on empiric cefazolin from post-trauma day 3 to post-trauma day 6 . She developed a fever $\left(T_{\max } 102.4\right)$ on post-trauma day 4 which subsided on post-trauma day 6 , at which point cefazolin was discontinued. During this time, a blood culture, a urine culture and two UAs were ordered, and came back clean. The patient remained afebrile until post-trauma day 10, at which point she spiked another low-grade fever. She was then put on ceftriaxone, and a chest x-ray and second round of cultures were ordered. The chest $\mathrm{x}$-ray showed no signs of pneumonia, the third UA was clean, a second urine 


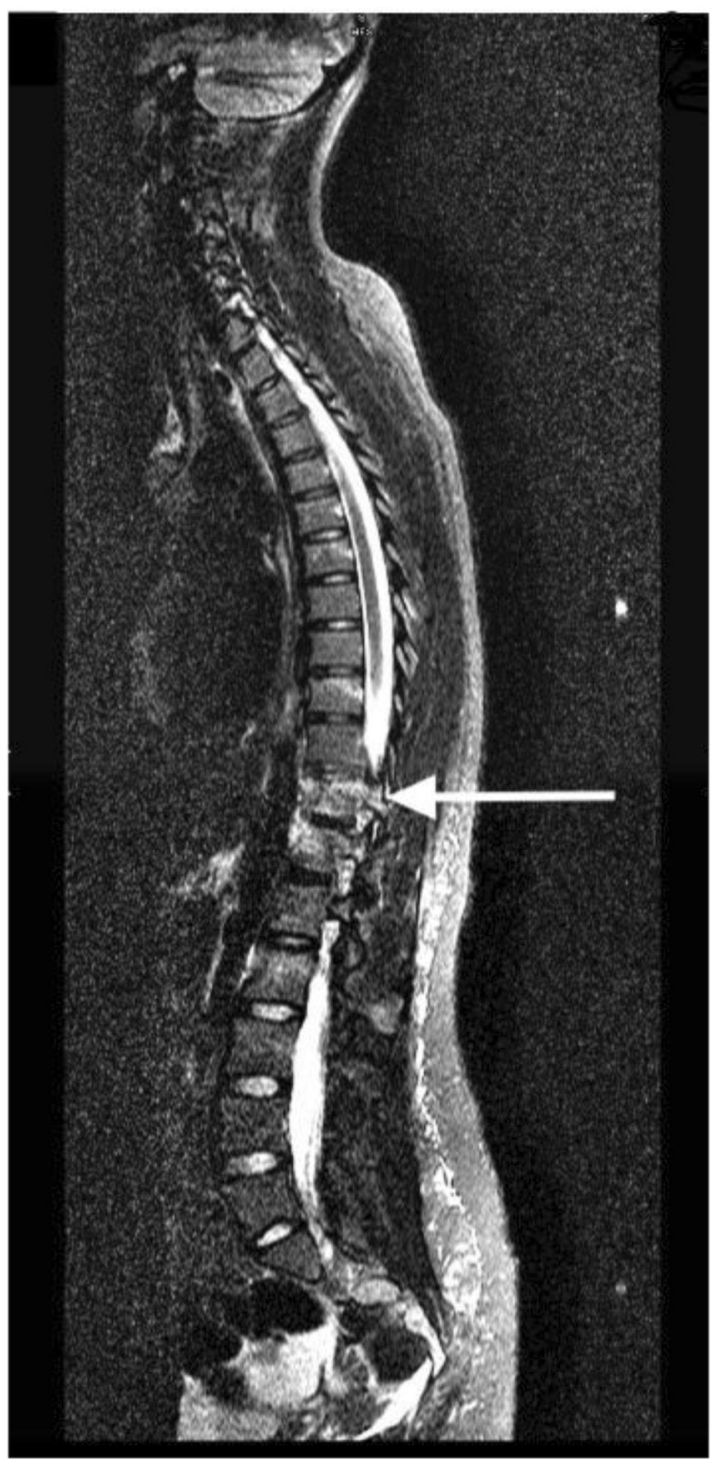

Fig. 1 "Traumatic spinal cord contusion at T10/T11 vertebral level is seen with surface hemorrhage along the spinal cord at the level of the contusion. The posterior longitudinal ligament and the interlaminar ligaments appear to be injured at T10/T11 vertebral level. There are fractures of T11 vertebral body with right T10/11 perched facet and facet articular process fractures, fractures of the right transverse process from T7 through T9 and T11 level. Additional vertebral body marrow edema without compression deformity is seen at T6, T7, T8, T9, and T10 vertebral levels. Rest of the cervical spine appears unremarkable".

culture was clean, and second and third blood cultures were clean. Her fever persisted and she was left on ceftriaxone. On post-trauma day 14 , patient developed ascending weakness, which was first reported by physical therapy, and hyperpyrexia, which was first reported by nursing. Both reports were made promptly and the patient was seen by physiatry. The review of systems was otherwise negative and the patient was transferred to PICU. Work up of new weakness by neurology included CSF studies (Table 1) and
Table 1 CSF study.

\begin{tabular}{|c|c|c|}
\hline Test & Ref. Range & Value \\
\hline Albumin, CSF & $0-35 \mathrm{mg} / \mathrm{dL}$ & $80(\mathrm{H})$ \\
\hline CSF Albumin Index & $0.0-9.0$ ratio & $24.6(\mathrm{H})$ \\
\hline CSF IgG Index & $0.28-0.66$ ratio & 0.63 \\
\hline CSF IgG Synthesis Rate & $<=8.0 \mathrm{mg} / \mathrm{d}$ & $33.6(\mathrm{H})$ \\
\hline CSF IgG/Albumin Ratio & $0.09-0.25$ ratio & $0.31(\mathrm{H})$ \\
\hline CSF Immunoglobulin G (IgG) & $0.0-6.0 \mathrm{mg} / \mathrm{dL}$ & $24.8(\mathrm{H})$ \\
\hline CSF Oligoclonal Bands & Negative & Negative \\
\hline Oligoclonal Bands Number & 0-1 Bands & 0 \\
\hline CSF Glucose & $45-80 \mathrm{mg} / \mathrm{dL}$ & $42 \mathrm{mg} / \mathrm{dL}$ \\
\hline CSF Protein & $15-40 \mathrm{mg} / \mathrm{dL}$ & $204(\mathrm{H})$ \\
\hline WBC CSF & $0-5 / \mathrm{uL}$ & $17(\mathrm{HH})$ \\
\hline RBC CSF & $0-0 / \mathrm{uL}$ & $1(\mathrm{H})$ \\
\hline CSF Cell Count Tube \# & Unknown & Tube \#3 \\
\hline CSF Color & Unknown & Colorless \\
\hline CSF Turbidity & Unknown & No Turbidity \\
\hline CSF Lymphocytes & $40-80 \%$ & $18(\mathrm{~L})$ \\
\hline Polys, CSF & $0-6 \%$ & $36(\mathrm{H})$ \\
\hline CSF Monocytes & $15-45 \%$ & $46(\mathrm{H})$ \\
\hline Number of Cells, CSF & Unknown & 100 \\
\hline CSF Comment (Cells Counted) & Unknown & 100 \\
\hline E. coli K1 PCR & - & - \\
\hline H. Influenza PCR & - & - \\
\hline Listeria PCR & - & - \\
\hline N. meningitidis PCR & - & - \\
\hline Strep. agalactiae PCR & - & - \\
\hline Strep. pneumo CSF PCR & - & - \\
\hline Cytomegalovirus PCR & - & - \\
\hline Enterovirus PCR & - & - \\
\hline HSV 1 PCR & - & - \\
\hline HSV 2 PCR & - & - \\
\hline Human Herpes Virus 6 PCR & - & - \\
\hline Parechovirus PCR & - & - \\
\hline Varicella-Zoster CSF PCR & - & - \\
\hline Crypto neoformans/gattii PCR & - & - \\
\hline
\end{tabular}

CSF analysis demonstrates elevated WBCs with few PMNs, elevated $\mathrm{IgG}, \mathrm{IgG}$ synthesis rate, and $\mathrm{IgG} /$ albumin ration consistent with diagnosis of transverse myelitis, although IgG index is within normal limits. CSF protein is significantly elevated making multiple sclerosis unlikely. In addition, no organisms were appreciated on Gram stain which further rules out infectious etiology.

repeat MRI (Fig. 2). Repeat MRI revealed "expansile hyperintensities consistent with longitudinally extensive transverse myelitis (TM) spanning the C2 to T11 levels." CSF study was negative for organisms but displayed moderate white cells with few neutrophils and only slightly decreased glucose. CSF analysis showed increased $\mathrm{IgG} /$ albumin ratio, elevated $\operatorname{IgG}$ synthesis rate, and elevated $\operatorname{IgG}$ confirming diagnosis of TM. Following diagnosis patient 


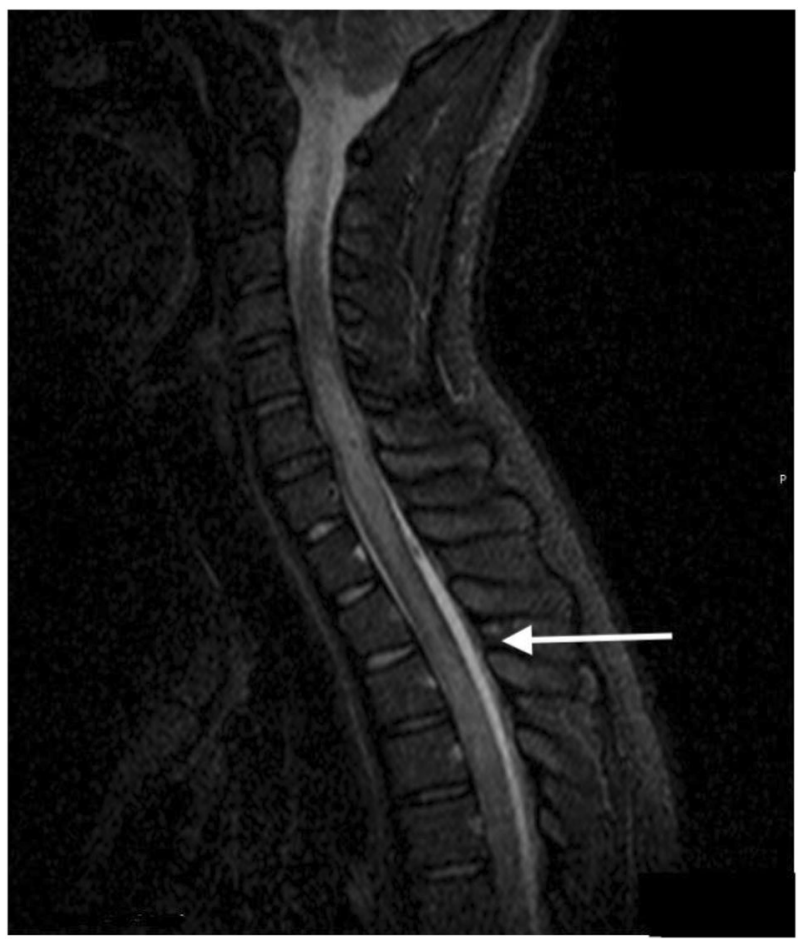

Fig. 2 "Interval development of extensive, expansile T2 cord hyperintensity without definite evidence of diffusion restriction extending from the inferior endplate of $\mathrm{C} 2$ to at least the level of T11, with patchy areas of cord enhancement. No epidural fluid collection or meningeal abnormality to suggest infection. Findings likely represent demyelination/myelitis. Again demonstrated are postsurgical changes from posterior rod and screw fixation from T9 through T12. Paramagnetic artifact from the metallic hardware somewhat limits evaluation of this region, but no large pseudomeningocele or epidural hemorrhage. Persistent bone marrow edema with mild anterior wedging demonstrated at T10 and T11 vertebrae is seen".

was given a 3-day course of high dose (1 g/day) methylprednisolone and IVIG, followed by oral prednisone. Her fever subsided and never recurred after day 2 of treatment with methylprednisolone (post-trauma day 16), however she was left on ceftriaxone until post-trauma day 24 . The patient was transferred back to the rehabilitation unit once stable to complete her rehabilitation course. Repeat examination graded her as a C6 AIS A SCI at this time. She tested at this level on post-trauma days 14,84 , and 99 . On post-trauma day 4 her upper extremity strength was rated $5 / 5$ throughout, lower extremity strength was rated $0 / 5$ throughout, and she exhibited sensory deficits up to T9 in an asymmetric distribution. On post-trauma day 14 her bilateral upper extremity strength was graded $3 / 5$ throughout, her bilateral lower extremity strength was graded $0 / 5$, and her sensory level was graded as C7. She was seen in clinic on posttrauma days 174 and 204 and scored 5/5 grades for bilateral upper extremity muscle strength with normal tone, improvement in performing ADLs, and only mild asymmetric abnormal sensation in a $\mathrm{T} 1$ distribution on the left.

\section{Discussion}

Traumatic spinal cord injuries are associated with many known complications and comorbidities. Evidence based guidelines are readily available to help direct spinal cord injury care and prevent these complications. DVT prophylaxis, respiratory management, pain management, and nutritional supplementation are proactive measures often taken with new spinal cord injury patients. Advances in preventative medical care have significantly improved the outcomes for individuals with spinal cord injuries [8].

Neurological deterioration is an unexpected complication that can impede recovery and rehabilitation efforts [9]. Typically due to mechanical instability following spine trauma, neurological deterioration can also result from other uncommon etiologies such as cord ischemia, syrinx, and inflammatory disorder. Transverse myelitis is one example of an inflammatory disorder that can occur following disruption of neural tissue. Timely diagnosis and treatment is important to help prevent worsening neurological status and delay of rehabilitation [1]. This necessitates all team members be able to recognize signs of deterioration they are most likely to come in contact with. Proper diagnosis of TM requires evidence of acute onset weakness and cord inflammation visible on MRI. In addition, CSF studies, $\mathrm{CBC}$, and MRI of the brain and spinal cord are required to rule out differential diagnoses such as syrinx, multiple sclerosis, meningitis, infectious myelopathy, cord ischemia, and systemic inflammatory disorders. Approximately $34 \%$ of TM patients attain nearly complete recovery, while the rest experience permanent deficits [3]. Rapid treatment with high dose steroids has significantly improved patient outcomes [1]. Quality of life for individuals with SCI is affected by secondary conditions that limit functional attainment [10]. Thus, rapid diagnosis and treatment of TM can significantly improve patients' longterm quality of life. In this case, prompt recognition of deterioration by multiple team members enabled this patient to make a complete functional recovery from her inflammatory injury.

\section{Conclusions}

Recognition of a second insult and administration of high dose steroids to reduce inflammation prevented further deficits and helped preserve quality of life in this case of TM following traumatic spinal cord injury. Proper recognition and treatment contributed to the patient making a functional recovery above the level of her traumatic injury. Despite the general stability of many rehabilitation patients, providers should remain vigilant for unexpected complications. All team members must effectively and promptly 
share information with each other so treatment decisions can be made.

In addition, providers should actively engage with other teams when these situations arise in order to provide optimal patient care.

Acknowledgements Imaging courtesy of Dr. Raghu Ramakrishnaiah ${ }^{2}$. This research was sponsored by AAP's MSSCE program.

\section{Compliance with ethical standards}

Conflict of interest A stipend in the amount of $\$ 4000$ was paid to Tyler M. Estes by the Association of Academic Physiatrists as part of the Medical Student Summer Clinical Externship Program. This program was entirely educational in nature and exerted no influence on the content of this case report.

Publisher's note Springer Nature remains neutral with regard to jurisdictional claims in published maps and institutional affiliations.

\section{References}

1. Transverse Myelitis Fact SheetNational Institute of Neurological Disorders and Stroke. https://www.ninds.nih.gov/Disorders/Pa tient-Caregiver-Education/Fact-Sheets/Transverse-Myelitis-FactSheet. Accessed August 11, 2018.

2. Ali A, Bareeqa SB, Riaz A, Ahmed SI, Shaikh MH, Ghauri MI Assessment of Clinical Outcomes in Patients Presenting with
Transverse Myelitis: a Tertiary Care Experience from a Developing Country. Cureus. 2019. https://doi.org/10.7759/cureus. 4342.

3. Bellinvia A, Pastò L, Razzolini L, et al. The clinical spectrum of anti-MOG associated acquired demyelinating disorders: three case-reports. Mult Scler Relat Disord. 2019;33:51-54. https://doi. org/10.1016/j.msard.2019.05.006

4. Abbas SA, Helou JE, Chalah MA. et al. Longitudinal extensive transverse myelitis in an immunocompetent older individual-a rare complication of varicella-zoster virus reactivation. Medicina. 2019;55:201. https://doi.org/10.3390/medicina55050201.

5. Ortega-Rosales A, Delgado-Torres N, Burneo-Rosales C. A rare neurological complication of tuberculosis: Transverse myelitis. IDCases. 2019;17. https://doi.org/10.1016/j.idcr.2019.e00564.

6. Sechi E, Shosha E, Williams JP, et al. Aquaporin-4 and MOG autoantibody discovery in idiopathic transverse myelitis epidemiology. Neurology. 2019. https://doi.org/10.1212/wnl. 0000000000007828.

7. Qin C, Chen B, Tao R, et al. The clinical value of complement proteins in differentiating AQP4-IgG-positive from MOG-IgGpositive neuromyelitis optica spectrum disorders. Mult Scler Relat Disord. 2019;35:1-4. https://doi.org/10.1016/j.msard.2019.06.035

8. Hagen EM Advances in pediatrics. https://www.ncbi.nlm.nih.gov/ pmc/articles/PMC4303786/\#B1. Published January 18, 2015. Accessed 8 Aug 2018.

9. Eric Belanger, MD, Claude Picard, MD, Daniel Lacerte, MD. "Subacute posttraumatic ascending myelopathy A report of three cases." J Neurosurg. 2000:294-9.

10. Barker RN, Kendall MD, Amsters DI, Pershouse KJ, Haines TP, Kuipers $\mathrm{P}$. The relationship between quality of life and disability across the lifespan for people with spinal cord injury. Spinal Cord. 2009;47:149-55. 